\title{
A Note on Caputo's Derivative Operator Interpretation in Economy
}

\author{
Hameed Ur Rehman $\left(\mathbb{D},{ }^{1,2}\right.$ Maslina Darus $\left(\mathbb{D},{ }^{1}\right.$ and Jamal Salah ${ }^{3}$ \\ ${ }^{1}$ School of Mathematical Sciences, Faculty of Science and Technology, Universiti Kebangsaan Malaysia, \\ Bangi 43600 Selangor Darul Ehsan, Malaysia \\ ${ }^{2}$ Center for Language and Foundation Studies, A' Sharqiyah University, Post Box No. 42, Post Code No. 400 Ibra, Oman \\ ${ }^{3}$ College of Applied and Health Sciences, A' Sharqiyah University, Post Box No. 42, Post Code No. 400 Ibra, Oman
}

Correspondence should be addressed to Maslina Darus; maslina@ukm.edu.my

Received 29 May 2018; Revised 26 August 2018; Accepted 5 September 2018; Published 1 October 2018

Academic Editor: Igor Andrianov

Copyright (C) 2018 Hameed Ur Rehman et al. This is an open access article distributed under the Creative Commons Attribution License, which permits unrestricted use, distribution, and reproduction in any medium, provided the original work is properly cited.

\begin{abstract}
We propound the economic idea in terms of fractional derivatives, which involves the modified Caputo's fractional derivative operator. The suggested economic interpretation is based on a generalization of average count and marginal value of economic indicators. We use the concepts of $T$ - indicators which analyses the economic performance with the presence of memory. The reaction of economic agents due to recurrence identical alteration is minimized by using the modified Caputo's derivative operator of order $\lambda$ instead of integer order derivative $n$. The two sides of Caputo's derivative are expressed by a brief time-line. The degree of attenuation is further depressed by involving the modified Caputo's operator.
\end{abstract}

\section{Introduction}

Fractional calculus is the field of Mathematical study that grows out of the traditional definition of calculus integral and derivative operators as the same way of fractional exponents is an outgrowth of exponents of integer value. The birth of fractional calculus occurred in a letter from G. F. A. de L'Hospital to G. W Leibniz in 1695 posing a possible question "what if the order of derivative $\left(d^{n} f / d t^{n}\right)$ such that $n=$ $1 / 2$ ", in his reply he wrote "it will lead to a paradox, from which one day useful consequences will be drawn"; for more details, refer to a book by M. Sen $[1$, p. 1] and an article by Machado [2]. The quoted conversation of the two famous mathematicians evoked interest among the researchers and the theory of noninteger orders was further developed, generalized, and formulated by famous mathematicians such as Riemann, Liouville, L Euler, Letnikov, Grunwald, Marchuad, Weyl, Riesz, Caputo, Abel, and others; see, for example, [36]. Fractional derivatives and fractional integrals have vast applications in the field of physics, mechanics, engineering, and biology $[7,8]$. In recent progress the fractional derivatives and fractional integrals are applied to narrate the financial process [9-11] and economic process with memory [12]. Fractional derivatives and fractional integration have been interpreted in many ways such as geometric interpretation [13-16], informatic interpretation [17], and economic interpretation [18]. Some of the modern definitions of fractional derivative and fractional integrals are enlisted in the next section.

\section{Modern Definitions of Fractional Calculus}

Definition 1. Riemann defined the fractional integral of order $\alpha>0$ as

$$
I_{a}^{\alpha} f(x):=\frac{1}{\Gamma(\alpha)} \int_{a}^{x}(x-t)^{\alpha-1} f(t) d t
$$

Definition 2. Riemann-Liouville defined the fractional derivative of order $n-1<\alpha \leq n(n \in N)$ as

$$
\begin{aligned}
D_{a}^{\alpha} f(x) & :=\frac{d^{n}}{d x^{n}} I_{a}^{n-\alpha} f(x) \\
& =\frac{1}{\Gamma(n-\alpha)} \frac{d^{n}}{d x^{n}} \int_{a}^{x}(x-t)^{n-\alpha-1} f(t) d t .
\end{aligned}
$$




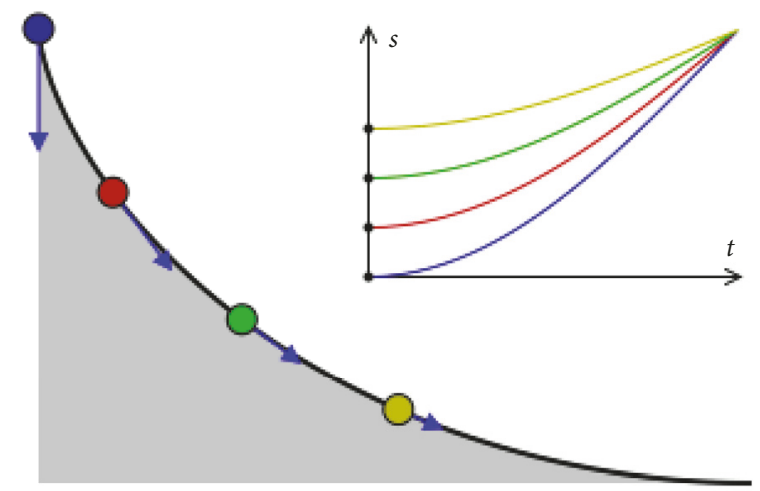

Figure 1: Four balls slide down a cycloid curve from different positions, but they arrive at the bottom at the same time, taken from [19].

Definition 3 (see [20]). Abel's integral equation of order $\alpha$ is given by

$$
I_{0}^{\alpha} f(x):=\frac{1}{\Gamma(\alpha)} \int_{a}^{x}(x-t)^{\alpha-1} y(x) d x .
$$

Remark 4. It is notable that Abel was the first one to use fractional calculus for $\alpha=1 / 2$ and solved the famous tautochrone problem $[20,21]$. For more details about the solution of problem and animated cycloid curve [19] see Figure 1. The total time of fall for $(f(y)=(2 / \sqrt{2 g})(d s / d y))$ is

$$
T=\int_{0}^{y_{o}}\left(y_{o}-y\right)^{-1 / 2} f(y) d y
$$

which is in fact the application of fractional integral of order $\alpha$, where the equation of cycloid is $d s / d y=$ $T(\sqrt{2 g} / \pi)(1 / \sqrt{y})$.

\section{Economic Meaning of Derivatives}

Before elaborating the economic meaning of fractional derivatives, we put up the economic concept of the standard derivative of the first order. In terms of economy the firstorder derivatives show the rapidness in alteration of economic indicators with respect to the investigated factor by assuming that other factors remain constant. The first-order derivative of the function of an indicator defines the marginal value of this indicator. The marginal value is the incremental value through the corresponding indicator per unit increase of the determining factor. In economic study, the principal marginal values of indicators are marginal product, margin utility, marginal profit, marginal cost, marginal revenue, marginal tendency to save and consume, marginal tax rate, marginal demand, and some others.

$$
\begin{aligned}
\text { Marginal Value }= & \text { Cost of Incremental Output } \\
& - \text { Cost of Incremental Input. }
\end{aligned}
$$

The economic processes is carried out by calculation of marginal and average value of indicators which are considered as a function of finding factors. According to the standard definition of average values and marginal values of indicators, a single value function as is supposed to be

$$
Y=Y(X)
$$

where $Y$ is the dependence economic indicator $Y$ by a factor $X$. The average value $A Y_{X}$ of the indicator $Y$ is given by

$$
A Y_{X}:=\frac{Y(X)}{X}
$$

The economic definition of marginal value $M Y_{X}$ of this indicator is given by the first derivative of (6) and is supposed to be a single-valued function.

$$
M Y_{X}:=\frac{d Y(X)}{d X}
$$

where $Y$ is the dependence economic indicator $Y$ by a factor $X$.

Mathematically if the dependence $Y=Y(X)$ is directly proportional to $(Y(X)=C . X)$ then marginal and average values are identical such that $M Y_{X}=A Y_{X}$.

Remark 5. Graphical analysis from a sample graph given in Figure 2 chosen from [22] shows the relation between marginal cost and average cost; also see [23].

(1) When the average curve is rising, then $M Y_{X}>A Y_{X}$ so product should decrease.

(2) When the average curve is at its minimum, then $M Y_{X}=A Y_{X}$, showing the peak of the average product.

(3) When the average curve is falling, then $M Y_{X}<A Y_{X}$ so product should increase.

In [18], Tarasova and Tarasov have stated the condition for the applicability of (8) that it defines the marginal value of an indicator under assumption that an indicator $Y$ must be expressed as a single valued function. In general this assumption is not valid [18]. Below are the two pair of examples in [18] of multivalued dependency of an indicator 


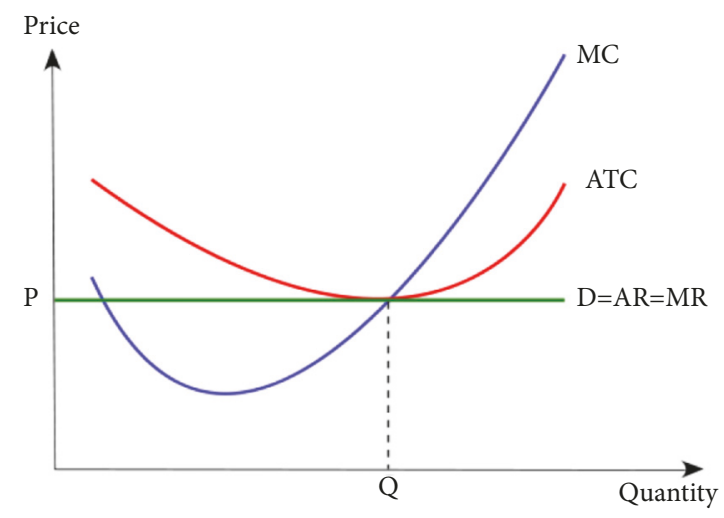

FIGURE 2: Three cost curves (average total cost, marginal cost, and marginal revenue) overlap.

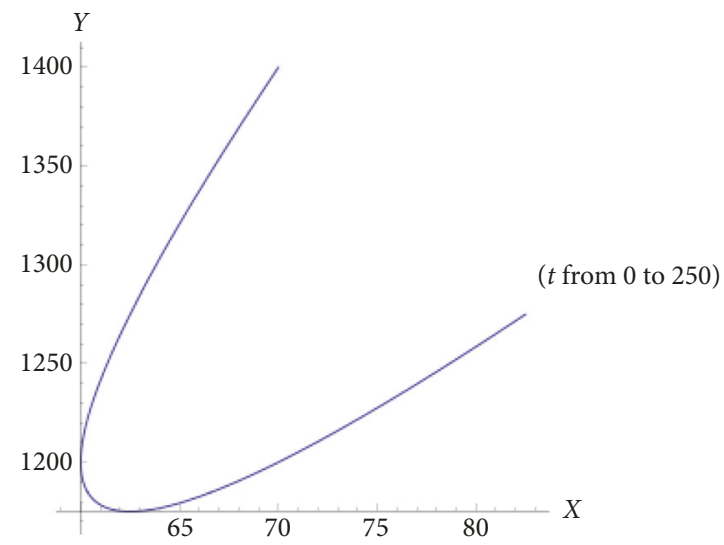

FIgURE 3: The graph in figure defined by equations (9), (10) shows the dependencies of an indicator $Y$ on a factor $X$.

$Y$ on a factor $X$, while involving the third variable the time $(t)$ and the indicator and factor are the function of $t$.

$$
\begin{aligned}
& X(t)=(0.001) t^{2}-(0.2) t+70, \\
& Y(t)=(0.01) t^{2}-(3.0) t+1400
\end{aligned}
$$

and second example

$$
\begin{aligned}
X(t)= & \left(8.2 \times 10^{-9}\right) t^{4}-\left(1.5 \times 10^{-5}\right) t^{3} \\
& +\left(5.4 \times 10^{-3}\right) t^{2}-(0.58) t+70, \\
Y(t)= & \left(7.5 \times 10^{-6}\right) t^{4}-\left(3.5 \times 10^{-3}\right) t^{3}+(0.51) t^{2} \\
& -(24.0) t+1700 .
\end{aligned}
$$

Note that the above expressions are the simplest appropriate polynomial approximation related to the "real change in the dollar - ruble exchange rate" [24].

Remark 6. Graph in Figure 3 clearly shows that the value of $X$ corresponds to more than one value of $Y$ in many cases, which violates the uniqueness of the function.

So, the formulas enlisted in (7) and (8) are not applicable to determine the average and marginal values of an indicator, as the dependence $Y$ on the factor $X$ is not one to one. So, for this type of economic analysis we need to consider the indicators and factors as a single valued function of time. And hence the indicator $Y=Y(t)$ on a factor $X=X(t)$, then applying to (7) and (8), we obtain

$$
\begin{aligned}
A Y_{X}(t) & =\frac{Y(t)}{X(t)} \\
M Y_{X}(t) & =\frac{d Y(t)}{d X(t)}
\end{aligned}
$$

Let the average value and marginal value for time $t=T$, so we have

$$
\begin{aligned}
A Y_{X}(T) & =\frac{Y(T)}{X(T)} \\
M Y_{X}(T) & =\left(\frac{d Y(t)}{d(t)} \cdot \frac{1}{d X(t) / d t}\right)_{t=T} \\
& =\frac{d Y(T)}{d(T)} \cdot \frac{1}{d X(T) / d T}
\end{aligned}
$$

provided $X(T) \neq 0$ and $d X(T) / d T \neq 0$.

The expressions (8) and (16) are equivalent and obey the chain rule if the dependence of $Y(t)$ on $X(t)$ can be expressed 


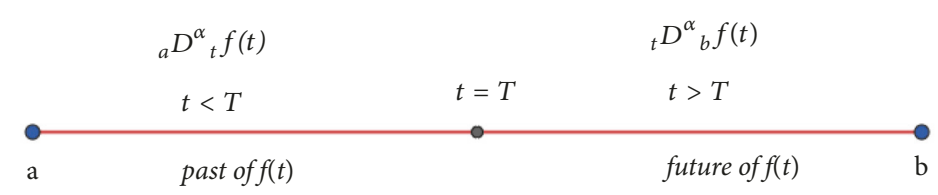

FIgURE 4: Action of derivative on three stages of functions.

as a single valued differentiable function $Y=Y(X)$. It is possible only if the function $X=X(t)$ is reversible, and hence (7) and (15) must be equivalent.

In the study of differential calculus, (16) can be considered as a generalized form of parametric derivative of indicator $Y=Y(t)$ by a factor $X=X(t)$ at time $t=T$, if $d X(T) / d T \neq 0$. It is important to note that (16) is the standard definition of the parametric derivative of first order if the function $X=$ $X(t)$ has an inverse in neighborhood of $t=T$ and first order derivative exists of the functions $X=X(t), Y=Y(t)$. Consequently, expressions (7) and (15) fail to be used for these dependencies; however, (15) and (16) can be used for parametric dependencies given by (9), (10) and/or (11), (12). So, we can say that (16) is associated with marginal indicator which concedes the first-order derivative as a growth of indicator $Y$ per unit increase of the factor $X$ at the given time point $t=T$.

It is worth mentioning that the violation of single valued property of the indicator function $Y(X)$ is due to the presence of memory in economic process in past [12, 18, 25]. Memory of economic agents influences the marginal indicators at the time $t=T$ and can depend on the changes of $Y(t)$ and $X(t)$ on finite time interval $(0, T)$. Average values and marginal values of indicators in (16) depend only on the given time $t=T$ and its diminutive neighborhood. Due to notable repeated similar alteration of the indicator $Y(t)$ and the factor $X(t)$ the economic agents can react on these identical changes. It is avoidable and can be applied only when the economic agents are in state of repression, and hence this approach cannot be always in practice for economic analysis. Mathematically this approach is because of applying integer order derivatives for economic analysis. So, the motivation is to involve a smaller degree to derive modified marginal values of an indicator function $Y(X)$ to put the past memory in a state of repression; see [18] for comparative modeling.

\section{Involvement of Modified Caputo's Derivatives Operator in Economy}

The Caputo's fractional derivative is one of the most used definitions of a fractional derivative along with other fractional calculus such as $(R-L),(G$. Letnikov) [26] and Caputo-type modification of the Erdély-Kober fractional derivatives. Here it is necessary to mention the Caputo-type modification of the Erdély-Kober fractional derivatives proposed by Luchko and Trujillo in 2007; see [27, 28] defined as follows.

Definition 7 (see[27]). Let $n-1<\delta \leq n, n \in \mathbb{N}$ and $m-1<\alpha \leq m, m \in \mathbb{N}$. The operators $\left({ }_{*} P_{\beta}^{\tau, \alpha} f\right)(x)$ and $\left({ }_{*} D_{\beta}^{\gamma, \delta} f\right)(x)$ are, respectively, called the left-hand and righthand sided Caputo-type modification of the Erdély-Kober fractional derivatives of orders $\alpha$ and $\delta$, respectively:

$$
\begin{aligned}
& \left({ }_{*} P_{\beta}^{\tau, \alpha} f\right)(x) \\
& :=\left(J_{\beta}^{\tau+\alpha, m-\alpha} \prod_{j=0}^{m-1}\left(\tau+j-\frac{1}{\beta} t \frac{d}{d t}\right) f\right)(x) \\
& \left({ }_{*} D_{\beta}^{\gamma, \delta} f\right)(x) \\
& :=\left(I_{\beta}^{\gamma+\delta, n-\delta} \prod_{k=0}^{n-1}\left(1+\gamma+k+\frac{1}{\beta} t \frac{d}{d t}\right) f\right)(x)
\end{aligned}
$$

where $J_{\beta}^{\tau, \alpha}$ and $I_{\beta}^{\gamma, \delta}$ of order $\alpha$ and $\delta$, respectively, called the left-hand and right-hand sided Erdély-Kober fractional integrals defined in [27].

The Caputo approach appears often while modeling applied problems by means of integrodifferential equations. Hence subsequent to our discussion in previous sections, the economic agents can react against identical changes in economic analysis and this is due to the use of integer order derivative. In continuation to this, the concept of noninteger derivative order $[3,6,29]$ is used in natural sciences to describe the process with memory. Recently, fractional order derivatives have been used to outline the financial processes $[12,18,25]$. However in this article we investigate the involvement of the modified Caputo's fractional operator [30, 31] for our results. We would like to highlight the two sides of the Caputo's time-fractional derivative by a brief time-line (see Figure 4). We use the concept of left-hand side of Caputo's derivative for our results, concept taken from $[1, \mathrm{p} .5]$.

Definition 8 (see[32]). “The left-hand side Caputo's fractional derivative of order $\alpha$ " is defined as follows:

$$
\begin{aligned}
& D_{0}^{\alpha} f(t) \\
& = \begin{cases}\frac{1}{\Gamma(n-\alpha)} \int_{0}^{t} \frac{f^{(n)}(\tau)}{(t-\tau)^{\alpha-n+1}} d \tau, & n-1<\alpha<n \in \mathcal{N} \\
\frac{d^{n}}{d t^{n}} f(t), & \alpha=n \in \mathscr{N}\end{cases}
\end{aligned}
$$

Caputo's derivative of $\operatorname{order} \alpha>0$ of the power functions:

$$
{ }_{0}^{C} D_{T}^{\alpha} t^{\beta}=\frac{\Gamma(\beta+1)}{\Gamma(\beta+1-\alpha)} t^{\beta-\alpha},
$$


Definition 9 (see [31]). Let $f \in A$ be analytic and normalized function. Then the modified Caputo's derivative operator of $f$ is defined by

$$
J_{\eta}^{\lambda} f(t)=\frac{\Gamma(2+\eta-\lambda)}{\Gamma(\eta-\lambda)} t^{\lambda-\eta} \int_{0}^{t} \frac{\Omega^{\eta} f(\zeta)}{(t-\zeta)^{\lambda-\eta+1}} d \zeta
$$

where $\eta \in \mathscr{R},(\eta-1)<\lambda<\eta<2$ and

$$
\begin{aligned}
\Omega^{\eta} f(\zeta) & =\Gamma(2-\eta) \zeta^{n} D_{\zeta}^{\eta} f(\zeta) \\
& =\frac{\Gamma(2-\eta) \Gamma(n+1)}{\Gamma(n-\eta+1)} \zeta^{n}
\end{aligned}
$$

Remark 10. Note that $J_{0}^{0} f(t)=f(T)$ and $J_{1}^{1} f(t)=T f^{\prime}(T)$.

Definition 11 (see [31]). "Special Case of modified Caputo's derivative operator" is defined as follows: let $\eta=0$ in Definition 2; we obtain

$$
J_{0}^{\lambda} f(t)=\frac{\Gamma(2-\lambda)}{\Gamma(-\lambda)} t^{\lambda} \int_{0}^{t} \frac{f(\tau)}{(t-\tau)^{\lambda+1}} d \tau, \quad-1<\lambda \leq 0 .
$$

Denoting the modified Caputo's fractional derivative operator by ${ }_{0}^{C} J_{\eta}^{\lambda} f(t)$ and omitting the constant term $(\Gamma(\eta-$ $\lambda) / \Gamma(2+\eta-\lambda)) t^{\eta-\lambda}$, from (21) by using (22) together with the relation of $\beta$-function and $\gamma$-function [33], we obtain the power functions of modified Caputo's fractional derivative operator of order $\lambda>0$ (same as in (20)); in the following form, see for details about below operator [30].

$$
{ }_{0}^{C} J_{\eta}^{\lambda} f(t)=\frac{(\Gamma(n+1))^{2} \Gamma(2-\eta) \Gamma(\eta-\lambda)}{\Gamma(n+\eta-\lambda+1) \Gamma(n-\eta+1)} t^{n-(\lambda-\eta),}
$$

where $(\eta-1<\lambda<\eta<2, \eta \in \mathscr{R}, t>0)$.

Remark 12. Note that the above power form of modified Caputo's fractional derivative corresponds to power function studied by Rubin \& Wenzel (1996); see [34], "that is accuracy in a memory task at time $t$ is given by $f(t)=a t^{-b}: a, b \in \mathbb{R}$ ".

For $f(t)=t,(24)$ becomes

$$
{ }_{0}^{C} J_{\eta, T}^{\lambda} t=\frac{T^{1-(\lambda-\eta)}}{(\eta-\lambda)(\eta-\lambda+1)} .
$$

Considering the effects of memory in economic process the generalized concept of marginal and average values of indicator in terms of Caputo's Left-hand side derivative with $(t \in[t ; T])$ is given by

$$
M Y_{X}(\alpha, T)=\frac{{ }_{0}^{C} D_{T}^{\alpha} Y(t)}{{ }_{0}^{C} D_{T}^{\alpha} X(t)}
$$

where $Y=Y(t)$ is economic indicator and $X=X(t)$ is determining factor. The above equation shows the economic $T$-indicator at time $t=T$, which confines the economic process with memory, where ${ }_{0}^{C} D_{T}^{\alpha}$ is the left-hand side derivative of order $\alpha \geq 0$ given in Definition 8 . The parameter $\alpha \geq 0$ demarcates the degree of depression in the memory about the changes of the indicator and factor on the interval $[0, T]$.

Economic research studies show that memory effect leads to abnormality in economic growth. The memory effect with declining order $0<\alpha<1$ results in slower growth of output compared with the standard model without memory; on the other hand the descending order of $0<\alpha<2$ can give faster growth output, for more details on economic growth model see [35].

For instance to count on changes in results of marginal values, we introduce the modified Caputo's fractional derivative of order $\lambda$ :

$$
\begin{aligned}
M Y_{X}(\lambda, \eta, T)= & \frac{{ }_{0}^{C} J_{\eta, T}^{\lambda} Y(t)}{{ }_{0}^{C} J_{\eta, T}^{\lambda} X(t)}, \\
& (\eta-1<\lambda<\eta<2, \eta \in \mathscr{R}, t>0),
\end{aligned}
$$

where ${ }_{0}^{C} J_{\eta, T}^{\lambda}$ is given by Definition 9 or (24).

Remark 13. Note for $\eta=0$ expression (27) coincides with the fractional order marginal value of (26) and for $\eta=1$ it coincides with marginal value of integer order as in (16).

It is also usable to replace $(X(t)=t)$ for economic indicator. So, using (27) the above expression becomes

$$
\begin{aligned}
& M Y_{X}(\lambda, \eta, T)=\frac{{ }_{0}^{C} J_{\eta, T}^{\lambda} Y(t)}{{ }_{0}^{C} J_{\eta, T}^{\lambda} t} \\
& =\frac{(\eta-\lambda)(\eta-\lambda+1)}{t^{1-(\lambda-\eta)}}\left({ }_{0}^{C} J_{\eta, T}^{\lambda} Y(t)\right) \\
& =(\eta-\lambda)(\eta-\lambda+1) T^{(\lambda-\eta)-1}\left({ }_{0}^{C} J_{\eta, T}^{\lambda} Y(t)\right),
\end{aligned}
$$

where $(\lambda-\eta)$ characterizes the degree of depression of the memory about the changes of indicators and factor on the interval $[0, T]$.

For the sake of numerical analysis of marginal values we apply the integer order derivative, Caputo's fractional order derivative, and modified Caputo's fractional order derivative, respectively, shown in forthcoming equations and tabulation. Manipulating (9), (10), and (16), we obtain the marginal value through integer order:

$$
M Y_{X}(1)=\left(\frac{0.02 t-3}{0.002 t-0.2}\right)_{t=1}=15.0505
$$

Using (9), (10), and (20), we get the marginal value through Caputo's fractional order:

$$
\begin{aligned}
& M Y_{X}\left(\frac{1}{2}, 1\right) \\
& =\left(\frac{0.0150451 t^{3 / 2}-(6 / \sqrt{\pi}) t^{1 / 2}+1400 / \sqrt{\pi} \sqrt{t}}{0.00150451 t^{3 / 2}-0.225676 t^{1 / 2}+70 / \sqrt{\pi} \sqrt{t}}\right)_{t=1} \\
& =20.0284
\end{aligned}
$$


TABLE 1: Marginal values for $\eta \in(1 / 2,1] \cup\{0\}$.

\begin{tabular}{lc}
\hline Depressive value & Marginal Value \\
\hline$\eta$ & $M Y_{X}\left(\frac{1}{2}, \eta, 1\right)$ \\
\hline 0 & 20.0284 \\
$33 / 64$ & 20.0288 \\
$17 / 32$ & 20.0292 \\
$9 / 16$ & 20.0305 \\
$5 / 8$ & 20.0336 \\
$11 / 16$ & 20.0382 \\
$3 / 4$ & 20.0455 \\
$13 / 16$ & 20.0579 \\
$7 / 8$ & 20.0832 \\
$15 / 16$ & 20.1616 \\
$31 / 32$ & 20.3265 \\
$63 / 64$ & 20.6906 \\
$127 / 128$ & 21.5932 \\
$255 / 256$ & 24.6707 \\
1 & 15.0403 \\
\hline
\end{tabular}

Making use of (9), (10), and (27), we get the marginal value through modified Caputo's operator of order $\eta$ :

$$
\begin{aligned}
M Y_{X}\left(\frac{1}{2}, \eta, 1\right) & =\frac{{ }_{0}^{C} J_{\eta, 1}^{1 / 2} Y(t)}{{ }_{0}^{C} J_{\eta, 1}^{1 / 2} X(t)}, \\
& \left(\eta-1<\frac{1}{2}<\eta<2, \eta \in \mathscr{R}, t=1>0\right),
\end{aligned}
$$

where

$$
\begin{aligned}
{ }_{0}^{C} J_{\eta, 1}^{1 / 2} Y(t)= & \frac{\Gamma(2-\eta) \Gamma(\eta-0.5)}{(25) \Gamma(\eta+2.5) \Gamma(3-\eta)} t^{\eta+1.5} \\
& -\frac{(3) \Gamma(\eta-0.5)}{\Gamma(\eta+1.5)} t^{\eta+0.5} \\
& +\frac{(1400) \Gamma(2-\eta)}{\Gamma(\eta+0.5) \Gamma(1-\eta)} t^{\eta-0.5}, \\
{ }_{0}^{C} J_{\eta, 1}^{1 / 2} X(t)= & \frac{\Gamma(2-\eta) \Gamma(\eta-0.5)}{(250) \Gamma(\eta+2.5) \Gamma(3-\eta)} t^{\eta+1.5} \\
& -\frac{\Gamma(\eta-0.5)}{(5) \Gamma(\eta+1.5)} t^{\eta+0.5} \\
& +\frac{(70) \Gamma(2-\eta)}{\Gamma(\eta+0.5) \Gamma(1-\eta)} t^{\eta-0.5} .
\end{aligned}
$$

Now for different choices of $\eta:(\eta-1<1 / 2<\eta<2)$ the marginal values by using the modified Caputo's operator in expression (31) are given in Table 1.

Remark 14. The sharpening behavior of the fractional values can be seen from (Table 1) as $\eta=1-\epsilon: \epsilon>0$ (the infinitesimal neighborhood before 1) results in maximum marginal value and on the other hand as $\eta=1 / 2+\epsilon: \epsilon>0$ (the infinitesimal neighborhood after $1 / 2$ ) results in minimum marginal value. Note that two integer values of $\eta=\{0,1\}$ where $\{0\}$ (is out of the interval) show anomalous character.

Some of the key notes are as follows:

(1) Equation (15) is the average values of indicator only for the values of the indicator and factor at times 0 and $T$.

(2) Equation (16) is the marginal values of indicator only for the values of the indicator and factor at $t=T$.

(3) The proposed economic indicator in (27) allows us to describe the dependence of economic process from all state of changes in a finite time interval $[0, T]$.

(4) The supplementary parameter $\eta \in \mathscr{R}$ lowers the rate of the past memory about the changes of indicator and factor on the interval $[0, T]$.

\section{Conclusion}

We used a modified Caputo's fractional operator towards the rectification of the indicator function $Y(X)$ by involving smaller degree $(\lambda-\eta)$ of forgetting memory. The influences of economic agents have been controlled comparative to the Caputo's fractional derivative of order $\alpha \geq 0$.

\section{Data Availability}

All data generated or analyzed during the study are included in the article.

\section{Disclosure}

The earlier version of this research article was presented in the "First Innovation Conference" on 21 December 2017, At A'Sharqiyah University Ibra, Sultanate of Oman.

\section{Conflicts of Interest}

The authors declare that there are no conflicts of interest. All the authors agreed with the content of the manuscript.

\section{Acknowledgments}

The work here is supported by UKM grant GUP-2017-064.

\section{References}

[1] M. Sen, Introduction to Fractional-Order Operators and Their Engineering Applications, 2014.

[2] J. A. T. Machado, A. M. S. F. Galhano, M. S. F. Alexandra, and J. J. Trujillo, "On development of fractional calculus during the last fifty years," Scientometrics, vol. 98, no. 1, pp. 577-582, 2014.

[3] S. G. Samko, A. A. Kilbas, and O. I. Marichev, Fractional Integrals and Derivatives, Theory and Applications, Gordon and Breach, Pennsylvania, Pa, USA, 1993. 
[4] V. S. Kiryakova, Generalized Fractional Calculus and Applications, CRC press, 1993.

[5] R. V. Mendes, Introduction to Fractional Calculus (based on lectures by R. Gorenflo, F. Mainardi and I. Podlubny), 2008.

[6] A. A. Kilbas, H. M. Srivastava, and J. J. Trujillo, "Theory and applications of fractional differential equations," North-Holland Mathematics Studies, vol. 204, 2006.

[7] T. F. Nonnenmacher and R. Metzler, Applications of Fractional Calculus Ideas to Biology, World Scientific, 1998.

[8] R. W. Ibrahim and M. Darus, "Infective disease processes based on fractional differential equation," in Proceedings of the $3 \mathrm{rd}$ International Conference on Mathematical Sciences, ICMS 2013, pp. 696-703, Malaysia, December 2013.

[9] E. Scalas, R. Gorenflo, and F. Mainardi, "Fractional calculus and continuous-time finance," Physica A: Statistical Mechanics and its Applications, vol. 284, no. 1-4, pp. 376-384, 2000.

[10] N. Laskin, "Fractional market dynamics," Physica A: Statistical Mechanics and its Applications, vol. 287, no. 3-4, pp. 482-492, 2000.

[11] F. Mainardi, M. Raberto, R. Gorenflo, and E. Scalas, "Fractional calculus and continuous-time finance. II: The waiting-time distribution," Physica A: Statistical Mechanics and its Applications, vol. 287, no. 3-4, pp. 468-481, 2000.

[12] V. V. Tarasova and V. E. Tarasov, "Marginal utility for economic processes with memory," Almanah Sovremennoj Nauki i Obrazovaniya [Almanac of Modern Science and Education], vol. 7, 2016.

[13] R. W. Ibrahim and M. Darus, "Differential operator generalized by fractional derivatives," Miskolc Mathematical Notes, vol. 12, no. 2, pp. 167-184, 2011.

[14] M. Darus and R. W. Ibrahim, "On classes of analytic functions containing generalization of integral operator," Journal of the Indonesian Mathematical Society, vol. 17, no. 1, pp. 29-38, 2011.

[15] J. Salah and M. Darus, "A note on generalized Mittag-Leffler function and applications," Far East Journal of Mathematical Sciences (FJMS), vol. 48, no. 1, pp. 33-46, 2011.

[16] J. Salah, "Fekete-szegö problems involving certain integral operator," International Journal of Mathematics Trends and Technology, vol. 7, no. 1, pp. 54-60, 2014.

[17] V. E. Tarasov, "Interpretation of fractional derivatives as reconstruction from sequence of integer derivatives," Fundamenta Informaticae, vol. 151, no. 1-4, pp. 431-442, 2017.

[18] V. V. Tarasova and V. E. Tarasov, "Economic Interpretation of Fractional Derivatives," Progress in Fractional Differentiation and Applications, vol. 3, no. 1, pp. 1-7, 2017.

[19] Wikipedia, Tautochrone curve - wikipedia, the free encyclopedia, 2017.

[20] E. Hairer and G. Wanner, Lanalyse au fil de l'histoire, vol. 10, Springer Science \& Business Media, 2001.

[21] P. Glaschke, Tautochrone and brachistochrone shape solutions for rocking rigid bodies, 2016.

[22] Wikimedia Commons, Costcurve - combined.svg - wikimedia commons, the free media repository, 2014.

[23] AmosWEB Encyclonomic WEB ${ }^{\star}$ pedia, Average Product and Marginal Product, LLC, 2000-2018.

[24] V. V. Tarasova and V. E. Tarasov, "On applicability of point price elasticity of demand to exchange trading on us dollar," Scientific Perspective, vol. 6, pp. 6-11, 2016.

[25] V. E. Tarasov and V. V. Tarasova, "Long and short memory in economics: fractional-order difference and differentiation,"
IRA-International Journal of Management and Social Sciences, vol. 5, no. 2, pp. 327-334, 2016.

[26] C. Li, D. Qian, and Y. Q. Chen, "On Riemann-Liouville and Caputo derivatives," Discrete Dynamics in Nature and Society, vol. 2011, Article ID 562494, 15 pages, 2011.

[27] Y. Luchko and J. J. Trujillo, "Caputo-type modification of the Erdélyi-Kober fractional derivative," Fractional Calculus and Applied Analysis, vol. 10, no. 3, pp. 249-267, 2007.

[28] V. Kiryakova and Y. Luchko, "Riemann-Liouville and caputo type multiple Erdélyi-Kober operators," Open Physics, vol. 11, no. 10, pp. 1314-1336, 2013.

[29] K. Oldham and J. Spanier, The Fractional Calculus Theory and Applications of Differentiation and Integration to Arbitrary Order, vol. 111, Elsevier, 1974.

[30] J. Salah, "A note on the modified caputo's fractional calculus derivative operator," International Journal of Pure and Applied Mathematics, vol. 109, no. 3, pp. 665-67, 2016.

[31] J. Salah and M. Darus, "A subclass of uniformly convex functions associated with a fractional calculus operator involving Caputo's fractional differentiation," Acta Universitatis Apulensis, no. 24, pp. 295-306, 2010.

[32] M. Caputo, "Linear models of dissipation whose Q is almost frequency independent-II," The Geophysical Journal of the Royal Astronomical Society, vol. 13, no. 5, pp. 529-539, 1967.

[33] Wikipedia, Beta function - wikipedia, the free encyclopedia, 2018, https://en.wikipedia.org/wiki/Beta_function.

[34] M. J. Kahana and M. Adler, Note on the Power Law of Forgetting, 2017.

[35] V. V. Tarasova and V. E Tarasov, "Fractional Dynamics of Natural Growth And Memory Effect in Economics," European Research, vol. 12, no. 23, pp. 30-37, 2016. 


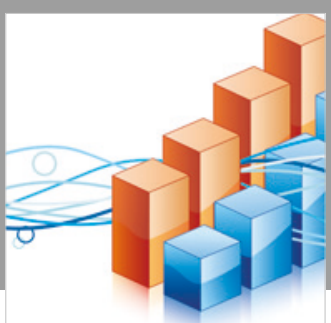

Advances in

Operations Research

\section{-n-m}
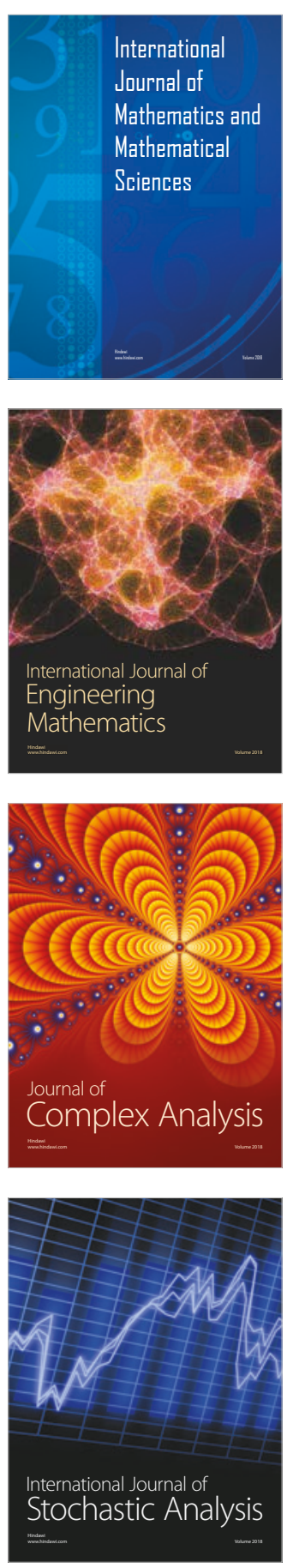
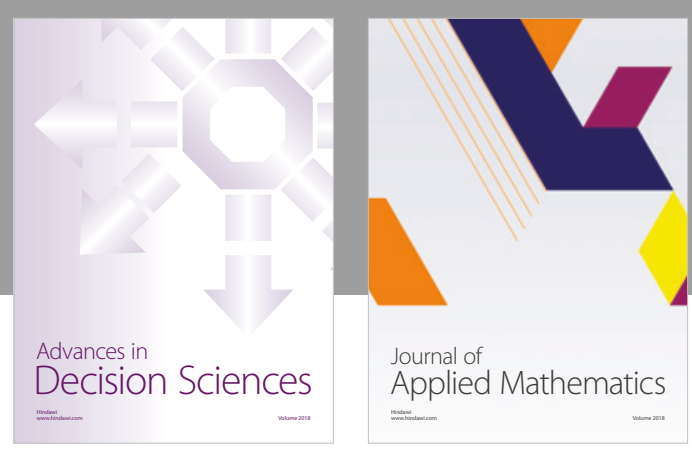

Journal of

Applied Mathematics
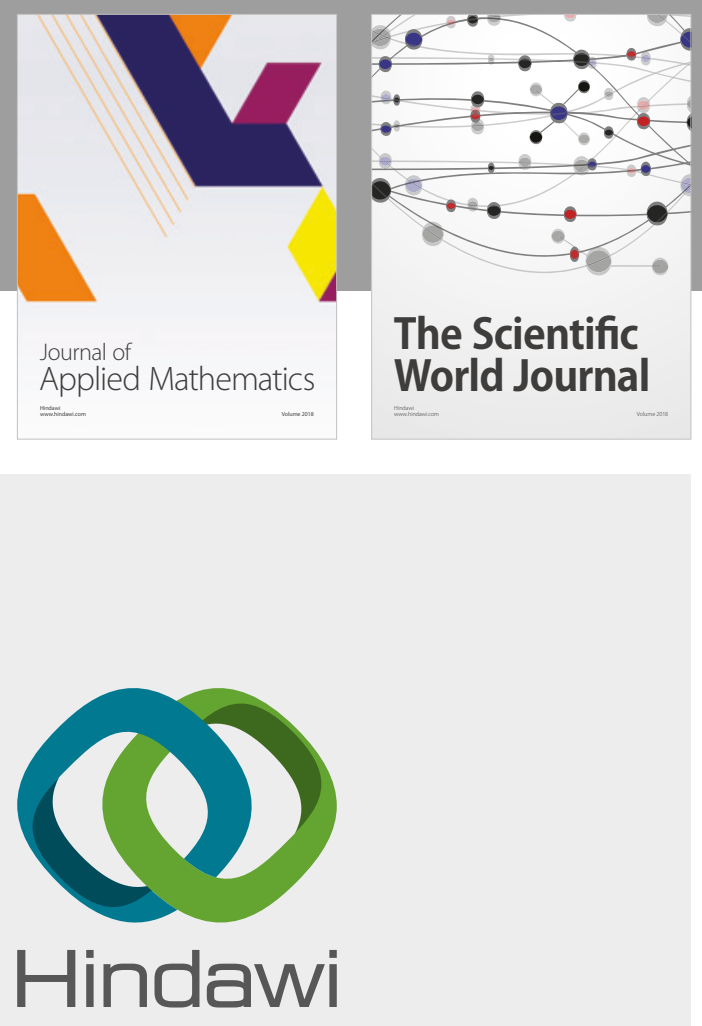

Submit your manuscripts at

www.hindawi.com

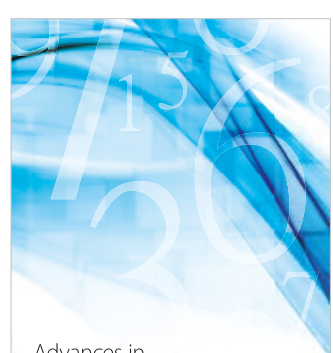

Advances in
Numerical Analysis
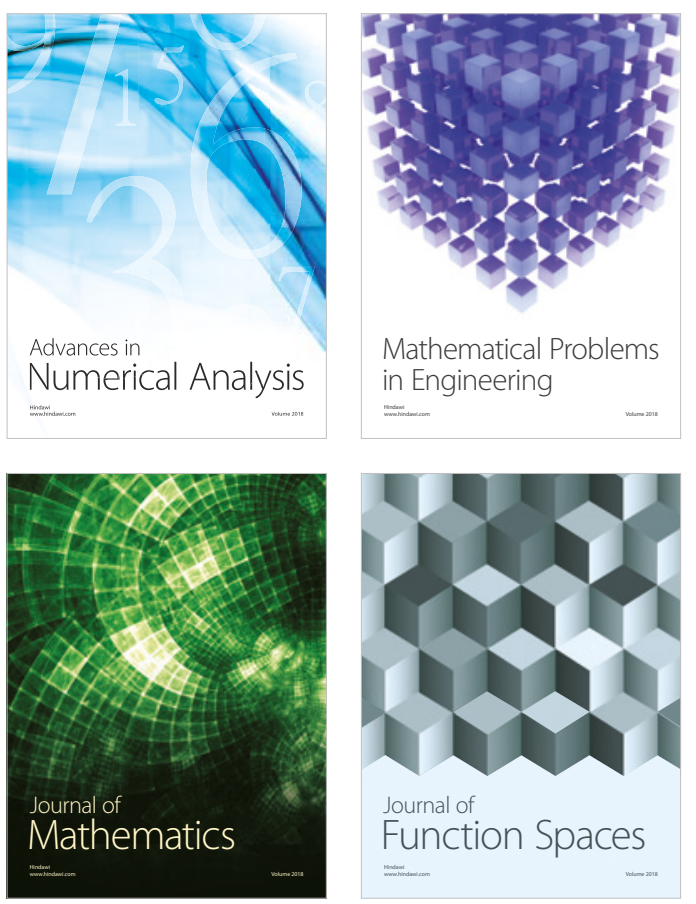

Mathematical Problems in Engineering

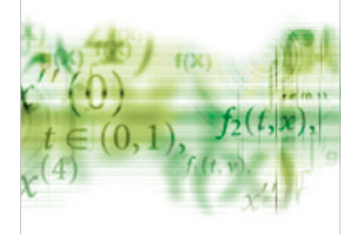

International Journal of

Differential Equations

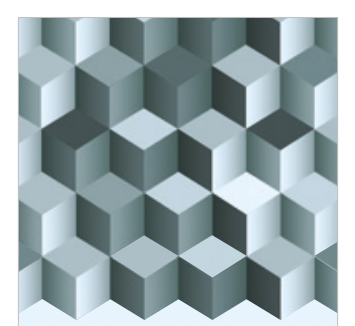

Journal of

Function Spaces

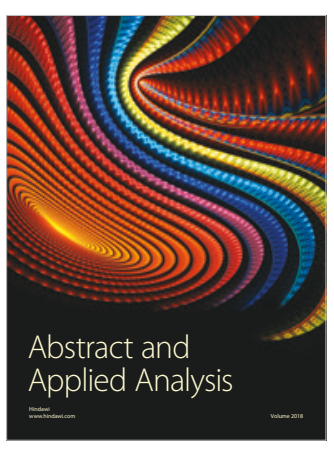

The Scientific

World Journal

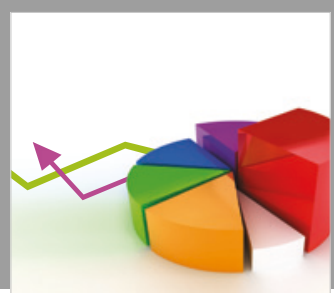

Journal of

Probability and Statistics
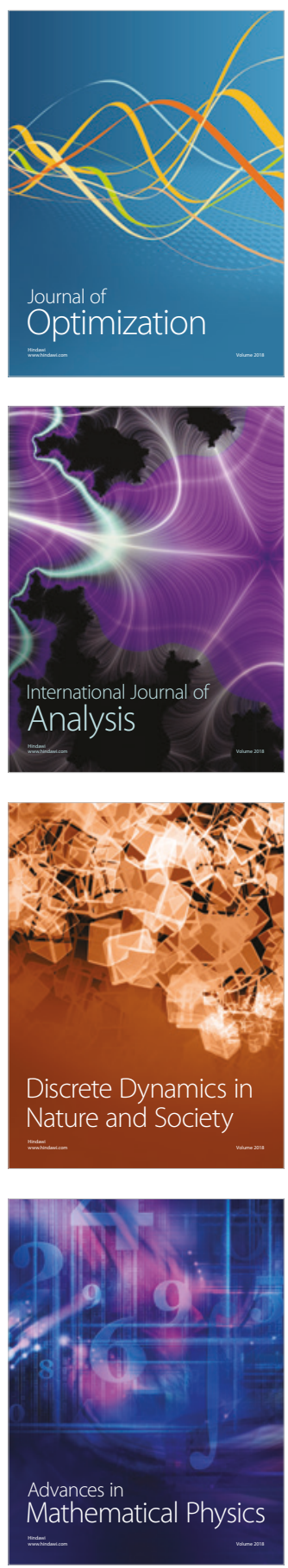